\title{
Dual codes of projective planes of order 25
}

\author{
Kelle L. Clark, Leslie D. Hatfield, Jennifer D. Key* and Harold N. Ward \\ Dedicated to Adriano Barloti on the occasion of his 80th birthday
}

\begin{abstract}
We determine improved bounds for the minimum weight of the dual code over $\mathbb{F}_{5}$ of any projective plane of order 25 and describe configurations that could give words of minimum weight.
\end{abstract}

\section{Introduction}

If $\Pi$ is a projective plane of order $n$ and $p$ is a prime dividing $n$, then the minimum weight of the dual $p$-ary code of $\Pi$ is not, in general, known, even in the desarguesian case. It is known that when the order of the plane is a prime $p$, the minimum weight is $2 p$ and words of this weight can be constructed from two distinct lines of the plane: see, for example, [1, Chapter 6]. For the binary dual code of desarguesian planes of even order $q=2^{m}$ the minimum weight is $q+2$ and the minimum words are the incidence vectors of the hyperovals, which always exist in the desarguesian planes. (See [10] for other results in the even case, and for when the plane has no hyperoval. In the latter case, again the minimum weight is not known except in some particular cases.) Some other results for $p$ odd are mentioned in Section 2. In particular, for the four planes of order 9, Key and de Resmini [11] proved that the minimum weight is 14 for the Hughes plane; and 15 for the desarguesian plane, $\Phi$, the translation (Hall) plane, $\Omega$, and the dual translation plane, $\Omega^{D}$.

In this paper we concentrate on the dual code of a projective plane of order 25 and prove the following theorem:

Theorem 1.1. If $\Pi$ is a projective plane of order 25 and $C$ is the code of $\Pi$ over $\mathbb{F}_{5}$, then the minimum weight $d^{\perp}$ of $C^{\perp}$ is either 42 or 44 , or $45 \leqslant d^{\perp} \leqslant 50$. If a Baer subplane is present, then the minimum weight is either 42,44 or 45 . In any case, if the minimum weight is 42 , then a minimum-weight word has support that is the union of two projective planes, $\pi_{1}$ and $\pi_{2}$, of order 4 that are totally disjoint and the (scaled) minimum-

* This work was supported by the DoD Multidisciplinary University Research Initiative (MURI) program administered by the Office of Naval Research under Grant N00014-00-10565, and NSF grant \#9730992. 
weight word is $\mathbf{v}^{\pi_{1}}-\mathbf{v}^{\pi_{2}}$. If the minimum weight is 44 then the support of a minimumweight word is the union of two complete 22-arcs that have eleven 2-secants in common. If the minimum weight is 45 then $\mathbf{v}^{\beta}-\mathbf{v}^{l}$, where $\beta$ is a Baer subplane of $\Pi$ and lis a line of $\Pi$ that is a line of the subplane, is a minimum-weight word.

(Two configurations in $\Pi$ are totally disjoint if they have no point or line in common.)

Corollary 1.2. The dual 5-ary code of the desarguesian projective plane $\mathrm{PG}_{2}\left(\mathbb{F}_{25}\right)$ has minimum weight 45.

In Section 2 we give the background results, definitions and notation, and in Section 3 we prove the main theorem through a series of lemmas and propositions.

\section{Background results and notation}

An incidence structure $\mathscr{D}=(\mathscr{P}, \mathscr{B}, \mathscr{I})$, with point set $\mathscr{P}$, block set $\mathscr{B}$ and incidence $\mathscr{I}$, is a $t$ - $(v, k, \lambda)$ design if $|\mathscr{P}|=v$, every block $B \in \mathscr{B}$ is incident with precisely $k$ points, and every $t$ distinct points are together incident with precisely $\lambda$ blocks. The number of blocks through a point of a $t \geqslant 1$-design is a constant, called the replication num$b e r$, and denoted by $r$. The order of the design is defined to be $r-\lambda$. An incidence structure $\mathscr{D}=(\mathscr{P}, \mathscr{B}, \mathscr{I})$ is a group divisible design if $\mathscr{P}$ is partitioned into point classes such that two points in the same class are incident with the same number $\lambda_{1}$ of blocks, and if any two points in distinct point classes are incident with the same number $\lambda_{2}$ of blocks.

If $\mathscr{S}$ is a set of points of $\mathscr{D}$ and if $B$ is a block of $\mathscr{D}$ that meets $\mathscr{S}$ in $m$ points, then $B$ will be called an $m$-secant to $\mathscr{S}$. The set $\mathscr{S}$ is an $\left(n_{1}, \ldots, n_{r}\right)$-set if $\mathscr{S}$ has $m$-secants if and only if $m \in\left\{n_{1}, \ldots, n_{r}\right\}$. The 1-secants are the tangents to $\mathscr{S}$.

The linear code $C$ of the design $\mathscr{D}$ over the finite field $F=\mathbb{F}_{p}$ is denoted $C_{p}(\mathscr{D})$, and is the vector space spanned by the incidence vectors of the blocks of $\mathscr{D}$ over $\mathbb{F}_{p}$. We denote the incidence vector of any subset $\mathscr{S}$ of $\mathscr{P}$ by $\mathbf{v}^{\mathscr{S}}$. We will always take $p$ to be a prime divisor of the order of $\mathscr{D}$ when looking at $C_{p}(\mathscr{D})$ : see [1, Theorem 2.4.1].

We view $C_{p}(\mathscr{D})$ as a subspace of $F^{\mathscr{P}}$, the full vector space of functions from $\mathscr{P}$ to $F$. Using the notation of functions, the value of $\mathbf{c} \in F^{\mathscr{P}}$ at a point $X \in \mathscr{P}$ is denoted $\mathbf{c}(X)$. The support set of $\mathbf{c}$ is the set of points $X$ in $\mathscr{P}$ for which $\mathbf{c}(X) \neq 0$, and the weight of $\mathbf{c}, \mathrm{wt}(\mathbf{c})$, is the cardinality of the support set of $\mathbf{c}$. The minimum weight of a code $C, d(C)=d$, is the smallest of all the non-zero weights of the codewords of $C$. The dual or orthogonal code $C^{\perp}$ of $C$ is the orthogonal space with respect to the standard inner product.

Let $\mathscr{D}$ be a $2-(v, k, \lambda)$ design with replication number $r$. Let $\mathscr{S}$ be the support set of a codeword in $C_{p}^{\perp}(\mathscr{D})$. For $i=0, \ldots,|\mathscr{S}|$, let $x_{i}$ denote the number of $i$-secants to $\mathscr{S}$. For $X$ a point in $\mathscr{S}, z_{i}(X)$ is the number of $i$-secants of $\mathscr{S}$ passing through $X$. It follows that $x_{1}=0$ and that $z_{1}=0$ for every point in $\mathscr{S}$. From counts on the $i$ secants $x_{i}$ of $\mathscr{Y}, 0 \leqslant i \leqslant k$, we have 


$$
\sum_{\substack{i=0 \\ i \neq 1}}^{k} x_{i}=b ; \quad \sum_{i=2}^{k} i x_{i}=s r ; \quad \sum_{i=2}^{k} i(i-1) x_{i}=s(s-1) \lambda
$$

and hence

$$
\sum_{i=3}^{k} i(i-2) x_{i}=s((s-1) \lambda-r)
$$

where the last equation is obtained from the previous two.

For a point $X$ in $\mathscr{S}$, with $z_{i}(X)=z_{i}$ (a shorthand we shall use whenever $X$ is obvious),

$$
\sum_{i=2}^{k} z_{i}=r ; \quad \sum_{i=2}^{k}(i-1) z_{i}=(s-1) \lambda .
$$

From these two equations we obtain the useful combination

$$
\sum_{i=2}^{k}(i-2) z_{i}=(s-1) \lambda-r
$$

Since the left-hand side of this equation is nonnegative, we have $(s-1) \lambda-r \geqslant 0$, i.e.

$$
s \geqslant \frac{r}{\lambda}+1
$$

for any word of $C^{\perp}$.

A $2-\left(n^{2}+n+1, n+1,1\right)$ design, for $n \geqslant 2$, is a finite projective plane of order $n$. We write $\mathrm{PG}_{2,1}\left(F_{q}\right)$ for the desarguesian projective plane, i.e. the design of points and 1-dimensional subspaces of the projective space $\mathrm{PG}_{2}\left(F_{q}\right)$. Further, $\mathrm{AG}_{2,1}\left(F_{q}\right)$ will denote the affine desarguesian plane of order $q$, i.e. the 2-design of points and 1-flats (cosets of vector subspaces of dimension one) in the affine geometry $\mathrm{AG}_{2}\left(F_{q}\right)$. A $k$ arc in a plane is a set of $k$ points, no three of which are collinear. A $k$-arc is said to be complete if it is not contained in a $(k+1)$-arc in the plane.

The current state of knowledge of the minimum weights of the dual codes of finite planes is summed up in the following results. The first is a special case of the designs from finite geometries and can be found discussed in [1, Theorem 5.7.9]:

Result 2.1. Let $C$ be the p-ary code of the desarguesian plane $\mathrm{PG}_{2,1}\left(\mathbb{F}_{q}\right)$ or $\mathrm{AG}_{2,1}\left(\mathbb{F}_{q}\right)$ where $q=p^{t}$ and $p$ is prime. Then the minimum weight $d^{\perp}$ of $C^{\perp}$ satisfies

$$
(q+p) \leqslant d^{\perp} \leqslant 2 q .
$$

Note that a similar range holds for any projective plane: if $\Pi$ is a plane of order $n$, $p$ is a prime, and $p \mid n$, the minimum weight $d^{\perp}$ of $C_{p}^{\perp}(\Pi)$ satisfies

$$
n+2 \leqslant d^{\perp} \leqslant 2 n .
$$


The lower bound is obtained by simply noticing that every one of the $n+1$ lines through a point in the support set of a word of minimum weight must meet the set again, and the upper bound follows since the vector $\mathbf{v}^{l}-\mathbf{v}^{m}$ is in $C_{p}^{\perp}(\Pi)$, where $l$ and $m$ are any two distinct lines of $\Pi$.

The next result can be found in [5, Corollary 4], and partly in [12]:

Result 2.2. Let $\Pi$ be a projective plane of odd order $n$, and let $p$ be a prime such that $p \mid n$. Then the minimum weight $d^{\perp}$ of $C_{p}^{\perp}(\Pi)$ satisfies $d^{\perp} \geqslant \frac{4}{3} n+2$. Further, if $p \geqslant 5$ then $d^{\perp} \geqslant \frac{3}{2} n+2$.

In addition the existence of a Baer subplane in a projective plane of square order gives us the following improved upper bound for $d^{\perp}$; see [12], [5].

Result 2.3. A projective plane of square order $q^{2}$ that contains a Baer subplane has words of weight $2 q^{2}-q$ in its $p$-ary dual code, where $p \mid q$.

In particular, this provides an upper bound for translation planes of square order; see [6], in which improved bounds for some translation planes were obtained:

Result 2.4. Let $\Pi$ be a projective translation plane of order $q^{m}$ and kernel containing $\mathbb{F}_{q}$, where $m=2$ or $3, q=p^{t}$, and $p$ is a prime. Then the dual code of the $p$-ary code of $\Pi$ has minimum weight at most $2 q^{m}-\left(q^{m-1}+q^{m-2}+\cdots+q\right)$. If $\Pi$ is desarguesian, this also holds for $m=4$.

Definition 2.5. For any vector $\mathbf{w} \in \mathbb{F}_{p}^{\mathscr{P}}$ with support set $\mathscr{S} \subseteq \mathscr{P}$ and $a \in \mathbb{F}_{p}^{*}$ define

$$
\mathscr{S}_{a}=\{X \in \mathscr{S} \mid \mathbf{w}(X)=a\}, \quad s_{a}=\left|\mathscr{S}_{a}\right|
$$

and

$$
\sigma(\mathbf{w})=\mid\left\{a \in \mathbb{F}_{p}^{*} \mid \text { there exists a point } Y \text { in } \mathscr{S} \text { with } \mathbf{w}(Y)=a\right\} \mid .
$$

The set $\mathscr{S}$ is a $j$-secant set if $\mathscr{S}$ has a 2 -secant, i.e. $x_{2} \neq 0$, and there exists an integer $j \geqslant 3$ such that $x_{i}=0$ for $2<i<j$ and $x_{j} \neq 0$.

The next result can be found in [5] and [4].

Result 2.6. Let $\mathscr{D}$ be a $2-(v, k, \lambda)$ design with replication number $r$ and order $n$. Let $\mathscr{S}$ be the support set of a non-zero word $\mathbf{w} \in C^{\perp}$, the dual code of the p-ary code $C_{p}(\mathscr{D})$, where $p$ is an odd prime and $p \mid n$. Suppose $|\mathscr{S}|=s \leqslant \frac{2 r}{\lambda}$. Then $z_{2}=z_{2}(X) \geqslant$ $2 r-(s-1) \lambda$ for every point $X$ in $\mathscr{S}$. Further, $\mathscr{S}$ is a j-secant set for some $j \geqslant 3$ and

(1) for any $X$ in $\mathscr{S}, z_{2}(X) \geqslant\left\lceil r \frac{j-1}{j-2}-\lambda \frac{s-1}{j-2}\right\rceil$;

(2) $s \geqslant \frac{\sigma(w)}{\sigma(w)+j-2}\left[\frac{r(j-1)}{\lambda}+1\right] \geqslant \frac{2}{j}\left(\frac{r(j-1)}{\lambda}+1\right)$.

Further, $\sigma(\mathbf{w})$ is even, and if $p>3$ and $j=3$, then $\sigma(\mathbf{w}) \geqslant 4$. 
In the next section we apply these results to the case where $\mathscr{D}$ is a projective plane of order 25. For these parameters, the inequalities in Result 2.6 become, writing $\sigma$ for $\sigma(\mathbf{w})$

$$
z_{2}(X) \geqslant\left\lceil\frac{26 j-s-25}{j-2}\right\rceil=26-\left\lfloor\frac{s-27}{j-2}\right\rfloor
$$

for any $X \in \mathscr{S}$, and

$$
s \geqslant \frac{\sigma}{\sigma+j-2}(26 j-25) .
$$

Using the notation of Definition 2.5 and Result 2.6, we have the following:

Lemma 2.7. Suppose that $p \nmid r$, as is the case for a projective plane. Then

$$
\sum_{a \in \mathbb{F}_{p}^{*}} a s_{a} \equiv 0 \quad(\bmod p)
$$

Proof. We have $\sum_{B \in \mathscr{B}} \mathbf{v}^{B}=r \mathbf{v}^{\mathscr{P}}$. Thus if $p \nmid r$, the all-one vector $\jmath=\mathbf{v}^{\mathscr{P}}$ is in $C_{p}(\mathscr{D})$, and the congruence follows from its orthogonality to the words of $C_{p}^{\perp}(\mathscr{D})$.

\section{Projective planes of order 25}

In what follows, let $\Pi$ be a projective plane of order 25 and set $C=C_{5}(\Pi)$. From Results 2.1 and 2.2 , the bounds on the minimum weight $d^{\perp}$ are $40 \leqslant d^{\perp} \leqslant 50$; or, from Result $2.3,40 \leqslant d^{\perp} \leqslant 45$ for planes containing a Baer subplane. In this section we investigate the structure of a support set $\mathscr{S}$ of a word $\mathbf{w}$ in $C^{\perp}$ having weight in this range. We first note that a constant word in $C^{\perp}$ must have size at least $(q+1)(p-1)+1=105$. From Result 2.6, $\sigma(\mathbf{w})=\sigma$ is either 2 or 4 , and $\mathscr{S}$ is a $j$ secant set for some $j \geqslant 3$. We now look at the different values of $\sigma$ and $j$ to determine the possible configurations of the set $\mathscr{S}$ of points in $\Pi$. If we fix $\sigma$ and take $s=$ $|\mathscr{S}| \leqslant 49$, then by using inequality (7) we can determine the largest value of $j$ for which $\mathscr{S}$ is a $j$-secant set.

Lemma 3.1. Let $\mathscr{S}$ be the support set of a word $\mathbf{w}$ of $C^{\perp}$. Suppose that $s=|\mathscr{S}| \leqslant 49$ and $\sigma=\sigma(\mathbf{w})$ is as defined in Definition 2.5, and suppose $\mathscr{S}$ is a j-secant set. Then

- if $\sigma=4$ then $j=3$ and $s \geqslant 43$;

- if $\sigma=2$ then

\begin{tabular}{||c||c|c|c|c|c|c|c|c|c|c||}
\hline$s$ & 49 & 48 & 47 & 46 & 45 & 44 & 43 & 42 & 41 & 40 \\
\hline$j$ & {$[4,16]$} & {$[4,12]$} & {$[4,10]$} & {$[4,8]$} & {$[4,7]$} & {$[4,6]$} & {$[4,5]$} & {$[4,5]$} & 4 & 4 \\
\hline
\end{tabular}

where $[4, n]$ denotes the range $4 \leqslant j \leqslant n$ for $j$. 
Recall that two configurations in $\Pi$ are called totally disjoint if they have no point or line in common. In what follows, we often refer to a point in $\mathscr{S}_{a}$ as an a, and specify a secant by listing its point in this notation. Thus a $\mathbf{1 3 3 3}$ (secant) is a 4-secant with one point in $\mathscr{S}_{1}$ and three points in $\mathscr{S}_{3}$.

Lemma 3.2. Let $\mathrm{w}$ be a word of $C^{\perp}$ and let $\mathscr{S}$ be the support set of $\mathrm{w}$. Let $\sigma(\mathbf{w})=4$ and suppose that for some $X$ in $\mathscr{S}, z_{2}(X)=53-s$, where $s=|\mathscr{S}|$. Then $s>45$.

Proof. Note first that inequality (6) implies that $53-s$ is the smallest possible value for $z_{2}$. By way of contradiction, assume that $43 \leqslant s \leqslant 45$. One has $z_{3}(X)=s-27$ and $z_{i}(X)=0$ for $i>3$ from Equation (4). On scaling we may assume that $X \in \mathscr{S}_{1}$. Since the 3-secants on $X$ have the form 113 or 122, the only secants on $X$ and a point of $\mathscr{S}_{4}$ are 2-secants, and $s_{4}=z_{2}(X)$. Let $X$ be on $t_{1} 113$ secants and $t_{2} 122$ secants. Then by counting the points in $\mathscr{S}_{1}, \mathscr{S}_{2}$, and $\mathscr{S}_{3}$, we obtain the equations

$$
s_{1}=t_{1}-1, \quad s_{2}=2 t_{2}, \quad s_{3}=t_{1} .
$$

Thus $s_{3}=s_{1}-1$, and as $t_{1}+t_{2}=s-27$, we have $s_{2}=2 s-52-2 s_{1}$. These counts hold for all $X$ in $\mathscr{S}_{1}$. Again as $53-s$ is the smallest possible $z_{2}, s_{a} \geqslant 53-s$ for all $a \in \mathbb{F}_{p}^{*}$. If we consider the 113 secants on a fixed 3, we see that $s_{1}$ has to be even. Similarly, the 122s on a fixed 2 show that $s_{1}+1 \leqslant s_{2}$. For $s=43$ there is no set of $s_{a}$ values satisfying these conditions at all. The other possibilities are:

$\begin{array}{ccccrc}\text { case } & s & s_{1} & s_{2} & s_{3} & s_{4} \\ 1 & 44 & 10 & 16 & 9 & 9 \\ 2 & 45 & 10 & 18 & 9 & 8 \\ 3 & 45 & 12 & 14 & 11 & 8\end{array}$

Case 1 is out, because rescaling $\mathbf{w}$ by 3 produces another word with $s_{4}=9$ whose other $s_{a}$ values no longer fit the parameter lists.

Since information on $z_{i}$ possibilities for $s=45$ and $z_{2} \leqslant 11$ will be needed here and later, we present it now. The values not given in a row are 0 , and the lists are those allowed by Equation (4):

$\begin{array}{rrrrr}z_{2} & z_{3} & z_{4} & z_{5} & z_{6} \\ 8 & 18 & & & \\ 9 & 16 & 1 & & \\ 10 & 14 & 2 & & \\ 10 & 15 & & 1 & \\ 11 & 12 & 3 & & \\ 11 & 13 & 1 & 1 & \\ 11 & 14 & & & 1\end{array}$

If $Y \in \mathscr{P}_{2}$ in Case 2 of table (8), then $z_{2}(Y) \leqslant 9$ (from $s_{3}=9$ ), and $z_{3}(Y) \geqslant 16$. But $Y$ is on ten 122 secants and at most four 244 secants, which is not enough. In Case 3, 
each $Y \in \mathscr{S}_{2}$ is on 12122 secants, so that $Y$ is on one further secant with another 2 and no 1 . As $z_{2}(Y) \leqslant 11$, the possibilities in (9) show that this additional secant is an $i$-secant with $i=4,5$, or 6 . It is thus either a 2233, making $z_{2}(Y) \leqslant 9$; a 22344, making $z_{2}(Y) \leqslant 10$; or a 224444. Then in any event, $z_{3}(Y) \geqslant 14$, so that $Y$ must be on at least two 244s. But there are only $\left(\begin{array}{l}8 \\ 2\end{array}\right)=28$ pairs of $4 \mathrm{~s}$ for the 14 possibilities for $Y$. Hence each $Y$ is on exactly two 244 s and therefore on a 224444 . At this point, however, there are too many pairs of $4 \mathrm{~s}$ required, and Case 3 is also not possible.

Proposition 3.3. Let $\mathscr{S}$ be the support set of a word $\mathbf{w}$ of $C^{\perp}$. Suppose that $s=$ $|\mathscr{S}| \leqslant 49$. Then either $s=42$ and $\mathscr{S}$ consists of two totally disjoint projective planes of order 4 , or $s \geqslant 44$.

Proof. First take $\sigma=2$ and $40 \leqslant s \leqslant 43$, so that $\mathscr{S}$ is a $j$-secant set where $j \in\{4,5\}$, by Lemma 3.1. We may scale $\mathrm{w}$ and assume that $\mathscr{S}=\mathscr{S}_{1} \cup \mathscr{S}_{4}$ without loss of generality. Suppose first that $\mathscr{S}$ is a 4-secant set. If $X$ is a point on a 4-secant with $X \in \mathscr{S}_{1}$, we have $s_{4} \geqslant z_{2}+2$, and similarly for $s_{1}$. Thus $s \geqslant 2\left\lceil\frac{79-s}{2}\right\rceil+4$, so that $s \geqslant 42$. If $s=42$, then $s_{1}=s_{4}=21$ and $z_{2} \geqslant 19$ for all points, while $z_{2}(X)=19$ if $X$ is on a 4-secant. In this case, with $X \in \mathscr{S}_{1}$, the remaining six lines through $X$ must have intersection with $\mathscr{S}$ completely in $\mathscr{S}_{1}$. Since these lines will all have to be 5secants at least, there would have to be at least 24 more points in $\mathscr{S}_{1}$, which is too many.

If $s=43$, then $z_{2} \geqslant 18$ and, as above, the existence of 4-secants gives that $s_{a} \geqslant 20$ for $a=1,4$. But by Lemma 2.7, $s_{1} \equiv s_{4} \equiv 4(\bmod 5)$, so that one of the $s_{a}$ must be 19 and the other 24. Thus $s \neq 43$.

Suppose now that $\mathscr{S}$ is a 5 -secant set, and again $\sigma=2, \mathscr{S}=\mathscr{S}_{1} \cup \mathscr{S}_{4}$. From Lemma 3.1, $s=42$ or 43. Suppose $s=42$. Then from inequality (6), $z_{2} \geqslant 21$ for any $X \in \mathscr{S}$. Thus $s_{1}=s_{4}=21$, forcing $z_{2}=21$. Then Equations (3) give us that $z_{2}(X)=$ $21, z_{5}(X)=5$, and $z_{i}(X)=0$ otherwise. It follows that any two points in $\mathscr{S}_{1}$ are together on exactly one 5 -secant of $\mathscr{S}$. Thus $\mathscr{S}_{1}$ is a $2-(21,5,1)$ design, i.e. a projective plane of order 4 . The set $\mathscr{S}_{4}$ is also a $2-(21,5,1)$ design and these designs do not share points or lines. Hence $\mathscr{S}_{1}$ and $\mathscr{S}_{4}$ are a pair of totally disjoint projective planes of order 4 embedded in $\Pi$.

If $s=43$ in the 5 -secant case, then $z_{2} \geqslant 21$ and $s_{a} \geqslant 21$. Once again, Lemma 2.7 gives the contradiction that the $s_{a}$ are 19 and 24 .

Consider now the case where $\sigma=4$, so that $\mathscr{S}$ is a 3 -secant set and $s \geqslant 43$, by Lemma 3.1. If $s=43$, then $z_{2}(X) \geqslant 10$ for all $X \in \mathscr{S}$, by inequality (6). Then each $s_{a} \geqslant 10$; as we cannot have $s_{a}>10$ for all $a$, we may scale to take $s_{4}=10$, making $z_{2}(X)=10$ for $X \in \mathscr{S}_{1}$. However, Lemma 3.2 rules out this situation.

This completes all the cases for the proposition, so we have $s \geqslant 44$.

To finish the proof of the main theorem, we need to consider the possibility that $s=44$ or $s=45$. We show first that $s=44$ can happen only if disjoint complete 22arcs are present, and we do this through two lemmas dealing with the different cases. 
In both lemmas we have $\mathscr{S}$ the support set of a word $w$ in $C_{5}^{\perp}(\Pi)$ of weight 44 , where $\Pi$ is a projective plane of order 25 .

Lemma 3.4. If $\mathscr{S}$ is a 4-secant set of size 44 then, on scaling, $s_{1}=s_{4}=22$, and, for every point $X \in \mathscr{S}, z_{2}=20, z_{4}=1$, and $z_{5}=5$.

Proof. From Lemma 3.1, $\sigma=2$, and from inequality (6), for any $X \in \mathscr{S}, z_{2} \geqslant 18$. As in the 4-secant argument in Proposition 3.3, it follows that (on scaling) $s_{a} \geqslant 20$ for $a=1,4$. By Lemma 2.7, it must be that $s_{1}=s_{4}=22$. For a point $X$ on a 4-secant, the only feasible solution is $z_{2}=20, z_{4}=1$ and $z_{5}=5$. The possibility of some of the points not being on 4-secants is easily ruled out by considering cases and invoking Equation (4), and so this set of parameters holds for all points of $\mathscr{S}$.

Lemma 3.5. If $\mathscr{S}$ has size 44, then it must be either a 4-secant set of the type described in Lemma 3.4 or else the union of two disjoint complete 22-arcs that have eleven 2secants in common. In the latter case, the parameters for $\mathscr{S}$ are $x_{0}=200, x_{2}=x_{3}=$ 220 , and $x_{4}=11$, and, for every point of $\mathscr{S}, z_{2}=10, z_{3}=15$ and $z_{4}=1$.

Proof. From Lemma 3.1, if $\mathscr{S}$ is not a 4-secant set, then $\mathscr{S}$ is a 3, 5 or 6-secant set. Suppose first that $\mathscr{S}$ is a 6-secant set. Then $\sigma=2$ from Lemma 3.1 and for any $X \in \mathscr{S}, z_{2} \geqslant 22$, from inequality (6). Thus $z_{2}=22$ for all points of $\mathscr{S}$, and $s_{1}=s_{4}=$ 22. For any point in $\mathscr{S}$, say $X \in \mathscr{S}_{1}$, the remaining four lines that are not 2-secants must be totally in $\mathscr{S}_{1}$, so there cannot be any 6-secants.

Now suppose that $\mathscr{S}$ is a 5 -secant set. From Lemma 3.1, $\sigma=2$, and from inequality (6), for any $X \in \mathscr{S}, z_{2} \geqslant 21$. Thus we can assume that either $s_{1}=21$ and $s_{4}=23$ or $s_{1}=s_{4}=22$. But Lemma 2.7 rules out the former case. If $s_{1}=s_{4}=22$, then if $z_{2}=21$ for some point $X \in \mathscr{S}_{4}$, the remaining five lines through $X$ must cover one point from $\mathscr{S}_{1}$ and 21 from $\mathscr{S}_{4}$ excluding $X$. The one point from $\mathscr{S}_{1}$ could then not have $z_{2} \geqslant 21$. Thus we must have $z_{2}=22$ for all points of $\mathscr{S}$. The only feasible solution to this is $z_{5}=3$ and $z_{10}=1$, for all points of $\mathscr{S}$. Counting point incidences with 5 -secants gives $44 \times 3=5 x_{5}$, which clearly has no solution.

Finally, suppose that $\mathscr{S}$ is a 3-secant set, so that $\sigma=4$. From inequality (6), for any $X \in \mathscr{S}, z_{2} \geqslant 9$. However, Lemma 3.2 excludes $z_{2}=9$; thus $s_{a} \geqslant 10$ for $a=$ $1,2,3,4$. We show that in fact $s_{a}$ cannot equal 10 .

Suppose (by scaling) that $s_{4}=10$; then for all points in $\mathscr{S}_{1}, z_{2}=10$, and it follows that $z_{3}=15$ and $z_{4}=1$. If $X \in \mathscr{S}_{1}$, the 4-secant through $X$ cannot contain a 4 , so it is either 1112 or 1333. If it is 1112, then on doing the point counts we arrive at $s_{2}=36-2 s_{1}$ and $s_{3}=s_{1}-3$. By the restrictions on the $s_{a}$, it must be that

- $s_{1}=13, s_{2}=11, s_{3}=10, s_{4}=10$.

On the other hand, if the secant is 1333 , then $s_{2}=32-2 s_{1}$ and $s_{3}=s_{1}+2$. This time there are two possibilities: one is the previous one scaled by 3 , and the other is

- $s_{1}=10, s_{2}=12, s_{3}=12, s_{4}=10$.

Hence we may assume we have one of these two sets of values; then all points of $\mathscr{S}_{1}$ 
are on the same type of 4-secant. If that secant is $\mathbf{1 1 1 2}$, then $s_{1}$ must be divisible by 3 ; so the case $s_{1}=13$ is out. When $s_{1}=10$, there will be 60122 secants. However, if we do the same argument for points in $\mathscr{S}_{4}$, we shall find ten 2224 secants. But then these two types of secants contain $60+30=90$ pairs of 2 s, and yet there are only $\left(\begin{array}{c}12 \\ 2\end{array}\right)=66$ available.

We can thus take $s_{a}=11$ for $1 \leqslant a \leqslant 4$. By Lemma $3.2, z_{2} \neq 9$ for all points in $\mathscr{S}$, and we get three possibilities for the secant counts through a point. Suppose that $X \in \mathscr{S}_{1}$. Then $X$ is on at most ten 113 secants and at most five 122 secants. For each of the secant counts for $X$, we can list the possibilities for the numbers of 3-secants of the two types and see whether the remaining points can be incorporated in the needed further secants. The results are these:

$\begin{array}{cccccccc}\text { case } & z_{2} & z_{3} & z_{4} & z_{5} & \text { \#113 } & \text { \# 122 } & \text { further secants } \\ 1 & 10 & 15 & 1 & 0 & 10 & 5 & \mathbf{1 2 3 4} \\ 2 & 11 & 13 & 2 & 0 & 8 & 5 & \mathbf{1 1 1 2}, \mathbf{1 3 3 3} \\ 3 & 11 & 14 & 0 & 1 & 10 & 4 & \mathbf{1 2 2 2 3} \\ 4 & 11 & 14 & 0 & 1 & 9 & 5 & \mathbf{1 1 2 3 3}\end{array}$

However, in Case 3, the 3 on the 5-secant would have $z_{2} \leqslant 8$ (the three $2 \mathrm{~s}$ on the 5 -secant are not on 2-secants with 3), and in Case 4, the 2 on the 5-secant would have $z_{2} \leqslant 9$. Neither of the resulting $z_{2}$ values allows a 5-secant, so these two cases are out. As $X$ is on five 122 secants in either remaining case, all 55 pairs of 2s appear on these secants. But the same argument applies to all the $\mathscr{S}_{a}$. That means there can be no aaab secants at all, and $z_{2}=10$ is the only possibility. All the 4-secants are 1234s, and each point of $\mathscr{S}$ is on exactly one of them. We have $x_{2}=x_{3}=220$ and $x_{4}=11$. Both $\mathscr{S}_{1} \cup \mathscr{S}_{4}$ and $\mathscr{S}_{2} \cup \mathscr{S}_{3}$ are complete 22-arcs in the plane and the eleven 4-secants are common secants to the two arcs. This completes the proof.

Proposition 3.6. If $C$ is the code over $\mathbb{F}_{5}$ of a projective plane $\Pi$ of order 25 with no complete 22-arcs then $C^{\perp}$ has no word of size 44 .

Proof. By Lemma 3.5, if $\Pi$ has no complete 22-arcs the support set $\mathscr{S}$ of a word of weight 44 must have the configuration described in Lemma 3.4. Let $\mathscr{S}=\mathscr{S}_{1} \cup \mathscr{S}_{4}$ as in Lemma 3.4, and let $\mathscr{T}=\mathscr{S}_{1}$.

We have $z_{2}=20, z_{4}=1$, and $z_{5}=5$ for any point of $\mathscr{S}$. Each 2-secant meets $\mathscr{T}$ in one point, each 4 -secant meets $\mathscr{T}$ in two points, and each 5 -secant meets $\mathscr{T}$ in five points. For $t \in \mathscr{T}$, let $t^{\prime}$ be the other point of $\mathscr{T}$ on the 4-secant through $t$; we have $\left(t^{\prime}\right)^{\prime}=t$. Let $\mathscr{F}$ be the collection of 5-subsets of $\mathscr{T}$ of the form $l \cap \mathscr{T}$, where $l$ is a 5secant meeting $\mathscr{T}$. If $t, u \in \mathscr{T}$ are distinct and $t^{\prime} \neq u$, then the line on $t$ and $u$ must be a 5 -secant; denote the corresponding member of $\mathscr{F}$ by $[t, u]$. It follows that $(\mathscr{T}, \mathscr{F})$ is a group divisible design in which the groups are the sets $\left\{t, t^{\prime}\right\}$. Moreover, if $F \in \mathscr{F}$, then because each point of $F$ is on four other members of $\mathscr{F}$, and $F$ and the resulting 20 members of $\mathscr{F}$ are all distinct, there is a unique $F^{\prime}$ in $\mathscr{F}$ that is disjoint from $F$. If $t \in F$, then as $t^{\prime}$ does not appear on any of these 20 members of $\mathscr{F}, t^{\prime}$ must be on $F^{\prime}$.

Now let $\mathscr{T}_{1}$ be a set of representatives of the pairs $\left\{t, t^{\prime}\right\}, t \in \mathscr{T}$, and let $\mathscr{F}_{1}$ be a set 
of representatives of the pairs $\left\{F, F^{\prime}\right\}$. Let $M$ be the $11 \times 11$ matrix with rows indexed by $\mathscr{F}_{1}$ and columns indexed by $\mathscr{T}_{1}$, in which the $(F, t)$ entry is given by

$$
\begin{aligned}
0 & \text { if } t, t^{\prime} \notin F \\
1 & \text { if } t \in F \\
-1 & \text { if } t^{\prime} \in F .
\end{aligned}
$$

Then each column of $M$ has five nonzero entries. Suppose $t$ and $u$ index different columns of $M$, and let $F=[t, u], G=\left[t^{\prime}, u\right]$. Then $F^{\prime}=\left[t^{\prime}, u^{\prime}\right]$ and $G^{\prime}=\left[t, u^{\prime}\right]$. The rows in which both the columns indexed by $t$ and $u$ have nonzero entries correspond to the pairs $\left\{F, F^{\prime}\right\}$ and $\left\{G, G^{\prime}\right\}$. It follows that the 2 by 2 submatrix for these two rows and columns is some scaling of

$$
\left[\begin{array}{cc}
1 & 1 \\
1 & -1
\end{array}\right]
$$

Consequently, the columns of $M$ are orthogonal, and $M^{T} M=5 I_{11}$. But that would mean $\operatorname{det} M=5^{11 / 2}$, which is impossible.

Thus there is no such word.

Proposition 3.7. Let $\mathbf{w}$ be a word of weight 45 in $C^{\perp}=C_{5}^{\perp}(\Pi)$, where $\Pi$ is a projective plane of order 25. Then $\mathbf{w}$ is a scalar multiple of $\mathbf{v}^{\beta}-\mathbf{v}^{l}$, where $\beta$ is a Baer subplane of $\Pi$ and $l$ is a line of $\Pi$ that is a line of the subplane.

Proof. Let $\mathscr{S}$ be the support set of $\mathbf{w}$ and let $\sigma=\sigma(\mathbf{w})$. First we consider $\sigma=2$, so that $\mathscr{S}$ is a $j$-secant set for some $j$ with $4 \leqslant j \leqslant 7$, by Lemma 3.1. Scale w to make $\mathscr{S}=\mathscr{S}_{1} \cup \mathscr{S}_{4}$. Inequality (6) implies that $z_{2}(X) \geqslant 17$ for any $X \in \mathscr{S}$, so that $s_{a} \geqslant 17$. As $s_{1}+s_{4} \equiv 0(\bmod 5)$ and $s_{1}-s_{4} \equiv 0(\bmod 5)$ by Lemma 2.7 , we may rescale again to assume that $s_{1}=25$ and $s_{4}=20$. Then for $X \in \mathscr{S}_{4}, z_{2}(X) \leqslant 20$. By inequality (6) again, $j=4$ or 5 . Suppose that $j=4$. As a 4-secant meets each $\mathscr{S}_{a}$ in two points, let $X \in \mathscr{Y}_{1}$ be on a 4-secant. Then $X$ is also on 172 -secants and one more secant with a point in $\mathscr{S}_{4}$. The remaining seven secants through $X$ must meet $\mathscr{S}$ in $\mathscr{S}_{1}$ alone, and so have sizes that are multiples of 5. By Equation (4), $\sum(i-2) z_{i}=18$, but these seven contribute at least 21 to the left side. Consequently $j=4$ is not possible.

With $j=5$, inequality (6) becomes $z_{2}(X) \geqslant 20$. Thus for all $X \in \mathscr{S}_{1}, z_{2}(X)=20$. As above, the remaining six secants on $X$ can only be 5-secants: $z_{5}(X)=6$ and $z_{i}(X)=0$ for $i \neq 2,5$. If $Y \in \mathscr{S}_{4}$, the secants on $Y$ and points of $\mathscr{S}_{1}$ are 2-secants. Thus $z_{2}(Y)=25$. The remaining secant on $Y$ must contain all the other points of $\mathscr{S}_{4}$. That is, $z_{20}(Y)=1$ and $z_{i}(Y)=0$ for $i \neq 5,20$. In other words, the points of $\mathscr{S}_{4}$ are collinear; let their line be $l$. The 25 points of $\mathscr{S}_{1}$ along with the 5 -secants now form an affine plane in $\Pi$. These 5 -secants can meet $l$ only in points outside of $\mathscr{S}_{4}$. It follows easily that with the addition of the six points of $l \backslash \mathscr{S}_{4}$ to $\mathscr{S}_{1}$ and the line $l$ to the 5secants, we create the Baer subplane needed in the statement of the proposition. 
Suppose $\sigma=4$, the value that must be ruled out. Then $j=3$, by Lemma 3.1, and for any $X \in \mathscr{S}, z_{2}(X) \geqslant 8$. Lemma 3.2 implies that $z_{2}(X) \geqslant 9$, in fact, so that $s_{a} \geqslant 9$ for all $a \in \mathbb{F}_{p}^{*}$. To begin with, suppose by scaling that $s_{4}=9$, making $z_{2}(X)=9$ for all $X \in \mathscr{S}_{1}$. Then $z_{3}(X)=16$ and $z_{4}(X)=1$, from (9). As in Lemma 3.5, the possible 4-secants on $X$ are 1112 and 1333, and we do the point counts in the two cases, with $X$ on $t_{1} 113$ secants and on $t_{2} 122$ secants, to find possible parameter values. If the 4secant is $\mathbf{1 1 1 2}$, we have

$$
s_{1}=t_{1}+3, \quad s_{2}=2 t_{2}+1, \quad s_{3}=t_{1}, \quad t_{1}+t_{2}=16 .
$$

If the 4 -secant is 1333 , then

$$
s_{1}=t_{1}+1, \quad s_{2}=2 t_{2}, \quad s_{3}=t_{1}+3, \quad t_{1}+t_{2}=16 .
$$

Since $s_{2}$ is odd for 1112 and even for 1333, all $X$ in $\mathscr{S}_{1}$ are on the same type of 4secant. In particular, $s_{1}$ must be divisible by 3 when the secants are 1112, and up to a further scaling, there is only one set of values:

- $s_{1}=12, s_{2}=15, s_{3}=9, s_{4}=9$.

If $Y \in \mathscr{S}_{2}$, then $z_{2}(Y)=9$ also, and $z_{3}(Y)=16$. But if $Y$ is on one of the 1112 secants, it is on at most nine 122s and at most four 244s, yielding too few 3-secants.

When the 4-secants are all 1333, $s_{1}+1 \leqslant s_{2}$ from the 122s on a 2 . If $s_{1}=9$, then $s_{2}=16$ and $s_{3}=11$; scaling the word by 4 produces a parameter list with $s_{4}=9$ that no longer fits the pattern. Two possibilities remain:

- $s_{1}=10, s_{2}=14, s_{3}=12, s_{4}=9$;

- $s_{1}=11, s_{2}=12, s_{3}=13, s_{4}=9$.

If $s_{1}=10$, then $z_{2}(Y)=10$ for $Y \in \mathscr{S}_{4}$ (as all secants on a 1 and a 4 are 2-secants), and $z_{3}(Y) \geqslant 14$ by (9). There are ten $1333 \mathrm{~s}$; they use 30 pairs of $3 \mathrm{~s}$ and leave $\left(\begin{array}{c}12 \\ 2\end{array}\right)-30=36$ pairs. Thus some $Y \in \mathscr{S}_{4}$ is on at most four 334 s and so on at least ten 244s. But there are not enough $4 \mathrm{~s}$ available for this.

Similarly, if $s_{1}=11$, there is $Y \in \mathscr{S}_{4}$ on at most five 334s. Since $Y$ is on at most eight 244s, $z_{3}(Y) \leqslant 13$. As $z_{2}(Y)=11$, (9) shows that $Y$ must be on a 4 -secant. There cannot be a 1 on it; and as all the pairs of $2 \mathrm{~s}$ are on the $122 \mathrm{~s}$, the only possibility is 3444. But $z_{3}(Y) \geqslant 12$, so that $Y$ is on at least seven 244s; but again, too many $4 \mathrm{~s}$ are needed.

Therefore $s_{a} \geqslant 10$ for all $a \in \mathbb{F}_{5}^{*}$. It cannot be that $s_{a} \geqslant 11$ for all $a$, for then three $s_{a}$ are 11 and one is 12 , and Lemma 2.7 excludes this. Scaling, we take $s_{4}=10$. Up to further scaling, Lemma 2.7 allows three possibilities:

- $s_{1}=11, s_{2}=13, s_{3}=11, s_{4}=10$;

- $s_{1}=12, s_{2}=11, s_{3}=12, s_{4}=10$;

- $s_{1}=15, s_{2}=10, s_{3}=10, s_{4}=10$.

Suppose that $s_{1}=15$. If $X \in \mathscr{S}_{1}$, then $X$ is on at most five 122s and at most ten 113s, 
making $z_{3}(X) \leqslant 15$. By $(9), z_{2}(X)=10$ and $z_{3}(X) \geqslant 14$. Then $X$ is on at least four $122 \mathrm{~s}$, so the 15 members of $\mathscr{S}_{1}$ require at least $60122 \mathrm{~s}$. As there are only 45 pairs of $2 \mathrm{~s}$ available, $s_{1}=15$ is ruled out.

Now let $s_{1}=12$, and use the argument finishing the proof of Lemma 3.2: for $X \in \mathscr{S}_{1}, X$ is on at most five 122s and at most 11 113s. The possibilities are

$\begin{array}{rcccccc}z_{2} & z_{3} & z_{4} & z_{5} & \# \mathbf{1 1 3} & \text { \# 122 } & \text { further secants } \\ 9 & 16 & 1 & 0 & 11 & 5 & \mathbf{1 2 3 4} \\ 10 & 15 & 0 & 1 & 11 & 4 & \mathbf{1 2 2 2 3} \\ 10 & 15 & 0 & 1 & 10 & 5 & \mathbf{1 1 2 3 3} \\ 10 & 14 & 2 & 0 & 9 & 5 & \mathbf{1 1 1 2}, \mathbf{1 3 3 3}\end{array}$

Regardless of the case, $X$ is collinear with at least five pairs of $2 \mathrm{~s}$, so that the secants on all the points of $\mathscr{S}_{1}$ account for at least 60 pairs (each such pair appears with just one 1 ). But there are only 55 pairs of $2 \mathrm{~s}$; thus $s_{1}=12$ is out.

Finally, suppose that $s_{1}=11$. Begin by rescaling $\mathbf{w}$ by 3 to take $s_{1}=13, s_{2}=10$, $s_{3}=11$, and $s_{4}=11$. Again we seek to reach a contradiction by counting pairs of $2 \mathrm{~s}$ as they appear on secants with points in $\mathscr{S}_{1}$. If the secant is a 122, that is the only secant this pair of $2 \mathrm{~s}$ is on. We do the same kind of secant analysis for a point $X$ in $\mathscr{S}_{1}$. There are quite a few, but only two in which $X$ is on at most three 122 secants:

$\begin{array}{cccccccc}z_{2} & z_{3} & z_{4} & z_{5} & z_{6} & \# 113 & \# 122 & \text { further secants } \\ 11 & 13 & 1 & 1 & 0 & 10 & 3 & \mathbf{1 1 1 2}, \mathbf{1 2 2 2 3} \\ 11 & 14 & 0 & 0 & 1 & 11 & 3 & \mathbf{1 1 2 2 2 2}\end{array}$

As the 3 on the 5 -secant in the first case has $z_{2} \leqslant 7$, this possibility is out. In the second, $X$ is collinear with nine pairs of $2 \mathrm{~s}$, six of them on the 6-secant. Since this secant contains two $1 \mathrm{~s}$, the count of pairs of $2 \mathrm{~s}$ from these $1 \mathrm{~s}$ is six apiece (their 122s give different pairs; by (9), no 1 can appear on two such 6-secants). Thus regardless of the secant pattern of $X$, we require at least $4 \times 13=52$ pairs of 2 s for the secant collinearities with points in $\mathscr{S}_{1}$, i.e. more than the 45 that are available.

Proof of theorem and corollary. The theorem is now proved, and for the corollary we note that if the plane $\Pi$ is desarguesian then complete 22-arcs do not exist; see [3], [9]. Thus 44 is not a possibility. Furthermore, $\Pi$ does not have subplanes of order 4; see, for example, [2]. Since $\Pi$ has Baer subplanes, the minimum weight is 45 .

Remarks. 1) In [11] it is noted that it is easy to show that in a plane of order 9 with a word of weight 15 in its dual ternary code, the word must have the same form that is established in Proposition 3.7.

2) All the known planes of order 25 have Baer subplanes; in particular, all translation planes of square order have Baer subplanes (see a new proof of this in [6]). Thus the minimum weight is at most 45 for the known planes.

3) No plane of order 25 has been shown to contain a subplane of order 4 , and some have been shown to not contain any; see [8].

4) The authors are unaware of any proofs of existence or non-existence of complete 
22-arcs, except in the desarguesian case. Even if a plane does have a 22-arc, it would need to have two 22 -arcs, $\mathscr{C}_{1}$ and $\mathscr{C}_{2}$, with the additional property that they share eleven secants, and of the remaining 220 secants to $\mathscr{C}_{1}$, say, 110 are external to $\mathscr{C}_{2}$ and 110 are tangents to $\mathscr{C}_{2}$.

5) It seems most likely that the minimum weight is 45 for all planes of order 25 . Note that the translation planes of order 25 were classified by Czerwinski and Oakden [7]. Most of these and some other (non-translation) planes of order 25 can be found at the web site: http://www.ces.clemson.edu/ $\sim \mathrm{keyj} / \mathrm{Key} /$ planes 25

\section{References}

[1] E. F. Assmus, Jr., J. D. Key, Designs and their codes. Cambridge Univ. Press 1992. MR 93j:51003 Zbl 0762.05001

[2] A. E. Brouwer, H. A. Wilbrink, Block designs. In: Handbook of incidence geometry, 349-382, North-Holland 1995. MR 97j:05018 Zbl 0823.51007

[3] J. M. Chao, H. Kaneta, Classical arcs in $\mathrm{PG}(r, q)$ for $23 \leqslant q \leqslant 29$. Discrete Math. 226 (2001), 377-385. MR 2002b:51005 Zbl 0977.51002

[4] K. L. Clark, Improved bounds for the minimum weight of the dual codes of some classes of designs. PhD Thesis, Clemson University 2000.

[5] K. L. Clark, J. D. Key, Geometric codes over fields of odd prime power order. In: Proceedings of the Thirtieth Southeastern International Conference on Combinatorics, Graph Theory, and Computing (Boca Raton, FL, 1999), volume 137, 177-186, 1999. MR 2000k:94053 Zbl 0996.94048

[6] K. L. Clark, J. D. Key, M. J. de Resmini, Dual codes of translation planes. European J. Combin. 23 (2002), 529-538. MR 1931937

[7] T. Czerwinski, D. Oakden, The translation planes of order twenty-five. J. Combin. Theory Ser. A 59 (1992), 193-217. MR 93c:51009 Zbl 0764.51004

[8] M. J. de Resmini. Private communication.

[9] G. Faina, F. Pambianco, On the spectrum of the values $k$ for which a complete $k$-cap in PG $(n, q)$ exists. J. Geom. 62 (1998), 84-98. MR 2000c:51008 Zbl 0917.51016

[10] J. D. Key, M. J. de Resmini, Small sets of even type and codewords. J. Geom. 61 (1998), 83-104. MR 98k:51024 Zbl 0910.51005

[11] J. D. Key, M. J. de Resmini, Ternary dual codes of the planes of order nine. J. Statist. Plann. Inference 95 (2001), 229-236. MR 2002b:94031 Zbl 0978.05018

[12] H. Sachar, The $\mathbb{F}_{p}$ span of the incidence matrix of a finite projective plane. Geom. Dedicata 8 (1979), 407-415. MR 81e:94020 Zbl 0419.51005

Received 19 December, 2002

K. L. Clark, Department of Mathematics, Queens University of Charlotte, Charlotte NC 28274, USA

Email: clarkc@queens.edu

L. D. Hatfield, H. N. Ward, Department of Mathematics, University of Virginia, Charlottesville VA 22904, USA

Email: \{ldw9e, hnw\}@virginia.edu

J. D. Key, Department of Mathematical Sciences, Clemson University, Clemson SC 29634, USA

Email: keyj@ces.clemson.edu 